\title{
Recubrimientos de aluminio-silicio realizados por deposición química de vapor en lecho fluidizado sobre el acero inoxidable AISI 316
}

\author{
Aluminum-Silicon Coatings Made by Chemical Vapor Deposition \\ in Fluidized Bed on Stainless Steel AISI 316
}

Fecha de Recepción: 2013-05-08

Fecha de Aprobación: 2013-06-18

\author{
José Luddey Marulanda Arevalo* \\ Francisco Javier Pérez Trujillo** \\ Aduljay Remolina Millán***
}

\section{Resumen}

Los recubrimientos de aluminio-silicio fueron depositados sobre el acero inoxidable AISI 316 mediante deposición química de vapor en lecho fluidizado (CVD-FBR), en el rango de temperaturas de 540 a $560{ }^{\circ} \mathrm{C}$, utilizando un lecho formado por 2,5 $\mathrm{g}$ de silicio y 7,5 $\mathrm{g}$ de aluminio en polvo, y $90 \mathrm{~g}$ de lecho inerte (Alúmina), el cual se hizo fluidizar con Ar. Como gases activadores se usó una mezcla de $\mathrm{HCl} / \mathrm{H}_{2}$, en relaciones de $1 / 10$ a $1 / 16$. Además, se varió el tiempo de deposición de los recubrimientos de 45 minutos a 1.5 horas, con una relación en volumen de $50 \%$ de gases activos y $50 \%$ de gases neutros. Se realizó una simulación termodinámica con la ayuda del programa informático Thermocalc, para obtener información de la posible

\section{Abstract}

The aluminum-silicon coatings were deposited on stainless steel AISI 316, by chemical vapor deposition, in fluidized bed reactors (CVDFBR), in the 540 to $560^{\circ} \mathrm{C}$ temperature range, using a bed formed by a $2.5 \mathrm{~g}$ silicon and $7.5 \mathrm{~g}$ aluminum powder and $90 \mathrm{~g}$ inert bed (alumina), which was fluidized with Ar. As activators were used a mixture of $\mathrm{HCl} / \mathrm{H}_{2}$ in ratios of $1 / 10$ to $1 / 16$. Furthermore, the coatings deposition time was varied between 45 minutes to 1.5 hours, with a volume ratio of $50 \%$ active gases, and $50 \%$ neutral gases. A thermodynamic simulation was conducted with the software Thermocalc for information about the possible composition and the amount of material deposited, for the selected conditions. Among the coatings are $\mathrm{FeAl}_{2} \mathrm{Si}$,

\footnotetext{
* Ph.D. Universidad Tecnológica de Pereira (Colombia).jlmarulanda@utp.edu.co

** Ph.D. Universidad Complutense de Madrid (España).

*** Ph.D. Universidad Pontificia Bolivariana de Bucaramanga (Colombia).
} 
composición y cantidad de material depositado, para las condiciones seleccionadas. En los recubrimientos se encuentran $\mathrm{FeAl}_{2} \mathrm{Si}, \mathrm{Fe}_{2} \mathrm{Al}_{5}$ y $\mathrm{FeAl}_{2}$. Los recubrimientos aluminio-silicio fueron tratados térmicamente, para mejorar sus propiedades mecánicas y su comportamiento frente a la oxidación, por la interdifusión de los elementos de aleación, ya que el tratamiento térmico hace que el aluminio difunda hacia el substrato, y el hierro difunda hacia la superficie del recubrimiento, logrando la transformación de los compuestos anteriores en $\mathrm{FeAl}, \mathrm{Al}_{2} \mathrm{FeSi}$, $\mathrm{Cr}_{3} \mathrm{Si}, \mathrm{AlCrFe}$ y $\mathrm{AlFeNi}$.

Palabras clave: Recubrimiento de aluminiosilicio, Deposición química de vapor, Acero inoxidable, Intermetálicos, Lecho fluidizado.
$\mathrm{FeAl}_{2}$ and $\mathrm{Fe}_{2} \mathrm{Al}_{5}$. Aluminum-silicon coatings were heat treated to improve their mechanical properties and behavior against oxidation. The inter diffusion of the alloying elements and the heat treatment causes the aluminum to diffuse into the substrate and the iron diffuse into the coating surface, achieving the above compounds transformation in $\mathrm{FeAl}, \mathrm{Al}_{2} \mathrm{FeSi}, \mathrm{Cr}_{3} \mathrm{Si}$, and AlFeNi AlCrFe.

Keywords: Aluminum-Silicon Coating, Chemical Vapor Deposition, Stainless Steel, Intermetallic, Fluidized bed. 


\section{INTRODUCCIÓN}

La deposición química en fase de vapor (Chemical Vapor Deposition -CVD-) consiste en la reacción química de una mezcla de gases precursores en el interior de un reactor, con el fin de depositar un recubrimiento sólido, en forma de capa delgada, sobre la superficie del substrato, y los subproductos de la reacción son evacuados hacia el exterior [1,2]. En esta técnica se utilizan las reacciones químicas de los gases precursores reactantes, que son activados por calentamiento, plasma y radiación electromagnética, entre otros, para formar una capa sólida y estable. Un aspecto importante de las reacciones en el proceso de CVD es la necesidad de activar los gases precursores para que se produzca la reacción, ya que en condiciones normales de operación, presión y temperatura, la velocidad de reacción suele ser baja, siendo el método empleado en la activación lo que diferencia los distintos tipos de deposición química de vapor, como la activación por temperatura, por plasma y por radiación electromagnética, entre otras; aunque en la mayoría de los casos es necesario una contribución de la temperatura para conseguir la activación total de los reactivos [3].

Por su versatilidad, la deposición química de vapor es un proceso muy versátil que permite diversas aplicaciones, como la deposición de películas metálicas y materiales cerámicos para la protección contra el desgaste, la corrosión y la oxidación a alta temperatura, para su uso en la industria aeronáutica, militar e ingenieril en general; también permite el depósito de capas amorfas, monocristalinas, policristalinas, con propiedades eléctricas especiales que permite su uso como dieléctricos, tal es el caso de los recubrimientos de silicio y germanio, utilizados en microelectrónica y optoelectrónica para la fabricación de dispositivos de conversión de energía ; además, se pueden fabricar fibras cerámicas y compuestos de matriz cerámica, como también materiales nanoestructurados para la industria electrónica y la biotecnología $[4,5]$.
El proceso CVD puede producirse a través de pirólisis, oxidación, reducción, hidrólisis o cualquier combinación de estas, y el tipo de reacción que se produce puede influir en la eficacia del sistema [6]. Los principales parámetros que deben tenerse en cuenta en la CVD son la temperatura, la presión, la concentración de gases reactantes y la cantidad total de corrientes de gases.

La deposición química de vapor por lecho fluidizado (CVD-FBR) es una variante de la técnica de deposición química de vapor, la cual combina los atributos de la activación térmica por calentamiento con el lecho fluidizado, ya que aprovecha las ventajas de los lechos fluidizados, como son la alta transferencia de masa y calor entre el gas, el lecho y las muestras inmersas dentro del reactor, permitiendo tener mayor uniformidad en la temperatura y una muy buena mezcla de los gases reactivos con las partículas fluidizadas; de este modo se logra un alto grado de reacción de todas las especies activadas en el lecho, ya que en la fluidización existe un excelente contacto entre las partículas sólidas y el medio de fluidización gaseoso [7-9].

La CVD-FBR ha sido usada para realizar tratamientos superficiales a fibras, partículas y polvos, y además se puede aplicar a una gran variedad de sistemas capa/substrato [10]; este proceso representa uno de los modos más eficaces de modificar las propiedades superficiales de micro o nanopolvos, ya que muchas de las propiedades de los polvos se relacionan firmemente con el estado superficial de las partículas que los constituyen, como la fluidez, la resistencia mecánica, la resistencia a la corrosión, la carga eléctrica, la sinterabilidad, etc. [11-13]. La investigación actual está dirigida hacia la protección de superficies expuestas al desgaste, la corrosión y la oxidación a altas temperaturas. Los materiales estructurales empleados en ingeniería deben presentar una elevada resistencia a la corrosión, la oxidación y el desgaste, junto con una alta tenacidad y buena 
adherencia, y el proceso CVD-FBR es efectivo para la formación de capas sobre substratos metálicos, mejorando la resistencia al desgaste, la corrosión y la oxidación a alta temperatura.

\section{Procedimiento Experimental}

Se usaron probetas del acero inoxidable austenítico AISI 316, las cuales fueron maquinadas (20 mm x $6 \mathrm{~mm}$ x $2 \mathrm{~mm}$ ) y lijadas desde papel esmeril N. ${ }^{\circ} 100$ hasta N. ${ }^{\circ} 600$, para luego limpiarlas en un baño de acetona por ultrasonido por un tiempo de 10 minutos. Los recubrimientos de $\mathrm{Al}-\mathrm{Si}$ fueron depositados usando el proceso de deposición química en fase vapor mediante lecho fluidizado (CVDFBR) a presión atmosférica. Los lechos usados fueron polvos de aluminio de $99,55 \%$ de pureza y polvos de silicio de $99,5 \%$ de pureza; como lecho inerte se usó polvo de alúmina $\left(\mathrm{Al}_{2} \mathrm{O}_{3}\right)$. La mezcla fue fluidizada con Ar como gas inerte (Ar de 99,999\%); como gas reactivo se usó el cloruro de hidrógeno ( $\mathrm{HCl}$ de 99,999\%), y como gas reductor, el hidrógeno $\left(\mathrm{H}_{2}\right.$ de 99,999\%). La morfología de las capas fueron analizados por SEM; la composición se estudió mediante EDAX, y la estructura, mediante DRX. El microscopio electrónico de barrido empleado fue un JEOL Mod. JM-6400, que tiene acoplado un sistema de análisis de EDAX. El análisis de difracción de rayos $\mathrm{X}$ se realizó empleando un equipo PHILIPS modelo MPD, y la fuente utilizada fue de cobre $\left(\mathrm{Cu}_{\mathrm{K} \alpha}=1,54056 \AA\right)$, empleando un voltaje anódico máximo de $45 \mathrm{kV}$ $\mathrm{y}$ una corriente de $40 \mathrm{~mA}$.

\section{Resultados}

Se presentan los resultados obtenidos para la codeposición de aluminio/silicio utilizando la técnica de CVD-FBR sobre el acero austenítico AISI316.Losparámetrosiniciales sedeterminaron mediante simulación termodinámica, con la ayuda del programa Thermo-Calc [14]. Estos parámetros fueron elegidos como guía inicial, para obtener las condiciones aproximadas de codeposición de aluminio-silicio y de esta forma realizar los primeros experimentos de aplicación del revestimiento. Mediante la simulación termodinámica se obtuvieron las posibles fases sólidas que se podrían encontrar durante el proceso de codeposición del aluminio-silicio sobre el acero inoxidable austenítico en función de la temperatura. La simulación se realizó entre 460 y $640{ }^{\circ} \mathrm{C}$, como se puede apreciar en la Fig. 1 , donde se muestran las fases sólidas presentes en el depósito para el acero AISI 316, como lo son el FeSi, el $\mathrm{Al}_{3} \mathrm{Ni}_{2}, \mathrm{Al}_{2} \mathrm{Fe}, \mathrm{Cr}_{5} \mathrm{Si}_{3}, \mathrm{BBC} \_\mathrm{A} 2$, $\mathrm{Mo}_{5} \mathrm{Si}_{3}$ y el $\mathrm{MoSi}_{2}$, donde el compuesto BCC_A2, está formado por $\mathrm{Fe}_{0,532} \mathrm{Al}_{0,263} \mathrm{Mn}_{0,193} \mathrm{Cr}_{0,005} \overline{\mathrm{Si}}_{0,005}$. $\mathrm{El} \mathrm{FeSi} \mathrm{es} \mathrm{el} \mathrm{compuesto} \mathrm{más} \mathrm{estable} \mathrm{en} \mathrm{todo} \mathrm{el}$ rango de temperatura; le siguen el $\mathrm{Al}_{3} \mathrm{Ni}_{2}$ y el $\mathrm{Cr}_{5} \mathrm{Si}$. El Al $\mathrm{Al}_{2} \mathrm{Fe}$ y el BBC_A2 están en pequeñas cantidades, y cuando se incrementa el BBC_A2, disminuye el $\mathrm{Al}_{2} \mathrm{Fe}$; por lo anterior, se esperaría que el recubrimiento tenga mayor cantidad de silicio que de aluminio, debido a que se favorece la formación de los compuestos de silicio, como se puede apreciar en la Fig. 1. A diferencia de la simulación, en el proceso de depósito del recubrimiento se favorece la formación de compuestos de aluminio debido a que se cuenta con mayor cantidad de aluminio que de silicio, como se explica más adelante.

En las pruebas preliminares de codeposición de aluminio-silicio en el reactor CVD-FBR se utilizó un lecho formado por un $5 \mathrm{~g}$ de aluminio en polvo, $5 \mathrm{~g}$ de silicio en polvo y $90 \mathrm{~g}$ de lecho inerte (Alúmina); se hizo fluidizar con argón al $61 \%$ en volumen, y como gases activadores se usó una mezcla de $37,1 \%$ de hidrógeno y $1,9 \%$ de ácido clorhídrico, en volumen, por un tiempo de 90 minutos a $580{ }^{\circ} \mathrm{C}$, ya que para esta temperatura, con ese tiempo de deposición y con estas cantidades de gases activos y neutros, se obtenían las mejores condiciones de deposición para el recubrimiento de aluminio. A partir de las condiciones anteriores se lograron buenos recubrimientos, de aproximadamente $10 \mu \mathrm{m}$ $(+/-4 \mu \mathrm{m})$, pero la cantidad de silicio que se incorpora al recubrimiento es inferior al $0,5 \%$. 


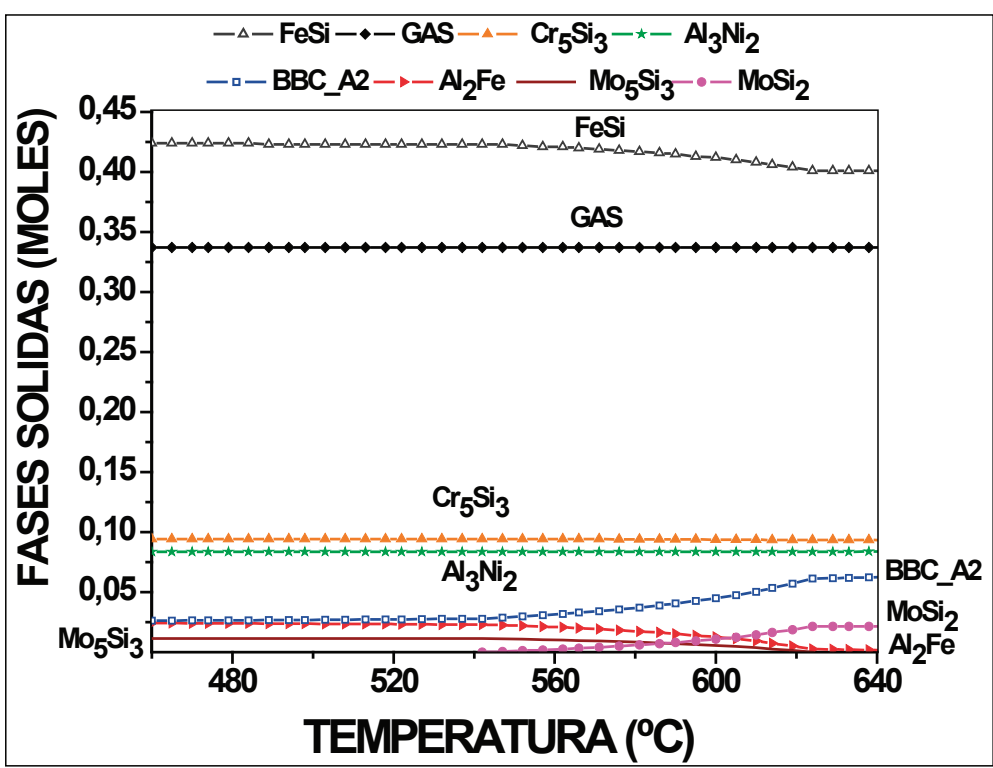

Fig. 1. Simulación termodinámica de la formación de las fases sólidas en la deposición de aluminio-silicio sobre el acero AISI 316.

Como la incorporación del silicio era mínima en la codeposición aluminio-silicio, se procedió a analizar la simulación termodinámica y a revisar las fuentes bibliográficas $[5,9,15$, 16], y se llegó a la determinación de bajar la temperatura de deposición a $540{ }^{\circ} \mathrm{C}$, ya que había mayor cantidad de $\mathrm{ClH}_{3} \mathrm{Si}$, y, por lo tanto, habría mayor posibilidad de que se depositara silicio; además, la activación térmica es buena, por lo que sería muy probable que se obtuvieran buenos recubrimientos aluminio-silicio. La cantidad de Al o Si que se deposita en la capa está controlada por la difusividad de las especies gaseosas y sólidas, sin embargo, la difusividad en la fase gaseosa es mucho mayor que en la fase sólida. Por lo tanto, el proceso de revestimiento es normalmente controlado por el crecimiento por difusión en estado sólido de las capas de aluminio o silicio $[17,18]$.

Se procedió a realizar los recubrimientos variando los gases activos, las cantidades de aluminio y silicio en el lecho y los tiempos de deposición, hasta obtener la codeposición aluminio-silicio. Los mejores recubrimientos de aluminio-silicio se obtuvieron cuando se realizó la deposición a $540{ }^{\circ} \mathrm{C}$, con una relación volumétrica de gases activos de $\mathrm{HCl} / \mathrm{H}_{2}: 1 / 15,3$, con 2,5 g de aluminio y 7,5 $\mathrm{g}$ de silicio en el lecho y una relación de $50 \%$ gases activos y $50 \%$ gases neutros. En la Fig. 2 se muestra la sección transversal de un recubrimiento de aluminio/ silicio sobre un sustrato de acero inoxidable austenítico AISI 316.

El recubrimiento aluminio-silicio sobre el acero AISI 316 tiene forma de conos, y su composición superficial en porcentaje en peso es $54,59 \%$ aluminio, $24,35 \%$ hierro, $8,05 \%$ níquel, $7,67 \%$ cromo, $3,62 \%$ silicio, $0,62 \%$ manganeso y $1,10 \%$ molibdeno, y se encuentra $\mathrm{FeAl}_{2} \mathrm{Si}$, $\mathrm{Fe}_{2} \mathrm{Al}_{5}$ y $\mathrm{FeAl}_{2}$, los cuales fueron determinados por DRX. Estos recubrimientos fueron tratados térmicamente, para mejorar sus propiedades mecánicas, a través de la interdifusión de los elementos de aleación. 


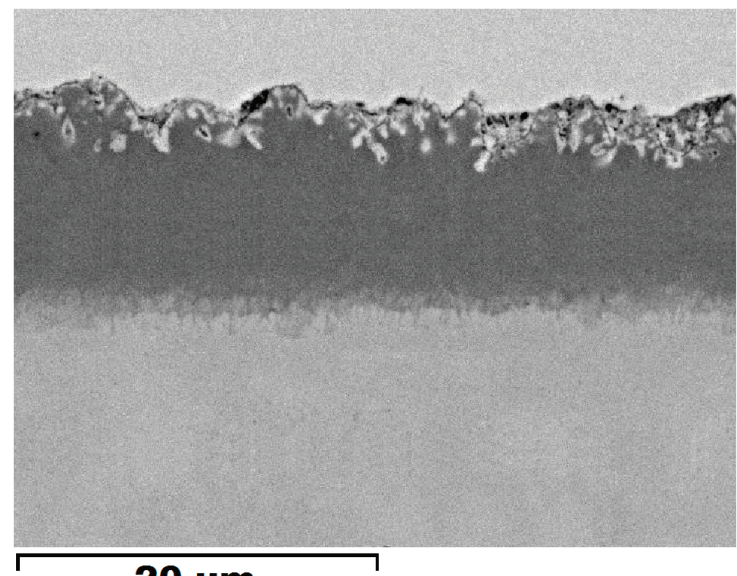

$20 \mu \mathrm{m}$

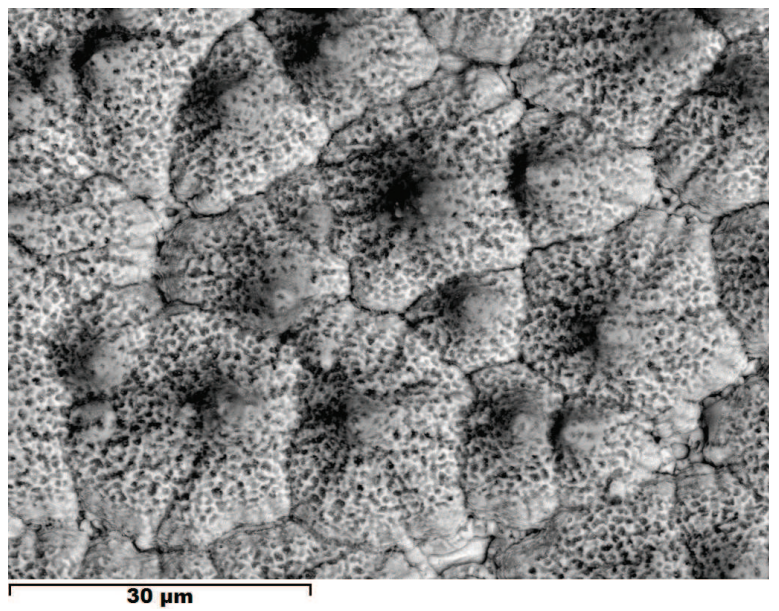

30 нm

Fig. 2. Imagen superficial del recubrimiento a la izquierda y corte transversal del recubrimiento aluminio-silicio a la derecha, sobre el acero AISI 316.

En la Fig. 3 se puede observar el corte trasversal del recubrimiento de aluminio-silicio con tratamiento térmico para el acero inoxidable austenítico, y al mismo tiempo se aprecia cómo varía la composición del revestimiento en función del espesor. Este revestimiento, tratado térmicamente y analizado por EDAX y DRX, está compuesto por $\mathrm{FeAl}, \mathrm{Al}_{2} \mathrm{FeSi}$, $\mathrm{Cr}_{3} \mathrm{Si}, \mathrm{AlCrFe}_{2}$ y $\mathrm{AlFeNi}$, en donde el $\mathrm{FeAl}$ está en mayor proporción en la parte externa de esta capa. El recubrimiento sin tratamiento térmico tiene mayor cantidad de aluminio que el recubrimiento tratado térmicamente; además, se aprecia el cambio de compuestos intermetálicos como $\mathrm{Al}_{5} \mathrm{Fe}_{2}, \mathrm{FeAl}_{2}, \mathrm{Al}_{2} \mathrm{FeSi}, \mathrm{Cr}_{3} \mathrm{Si}, \mathrm{AlCrFe}_{2}$ y $\mathrm{Al}_{5} \mathrm{FeNi}$. Después del tratamiento térmico desaparece el $\mathrm{Al}_{5} \mathrm{Fe}_{2}$ y se forma el FeAl; además se observó una disminución en el porcentaje de aluminio y un aumento en los porcentajes de hierro, cromo y níquel; por lo tanto, las fases que tienen estos elementos se vuelven más estables.
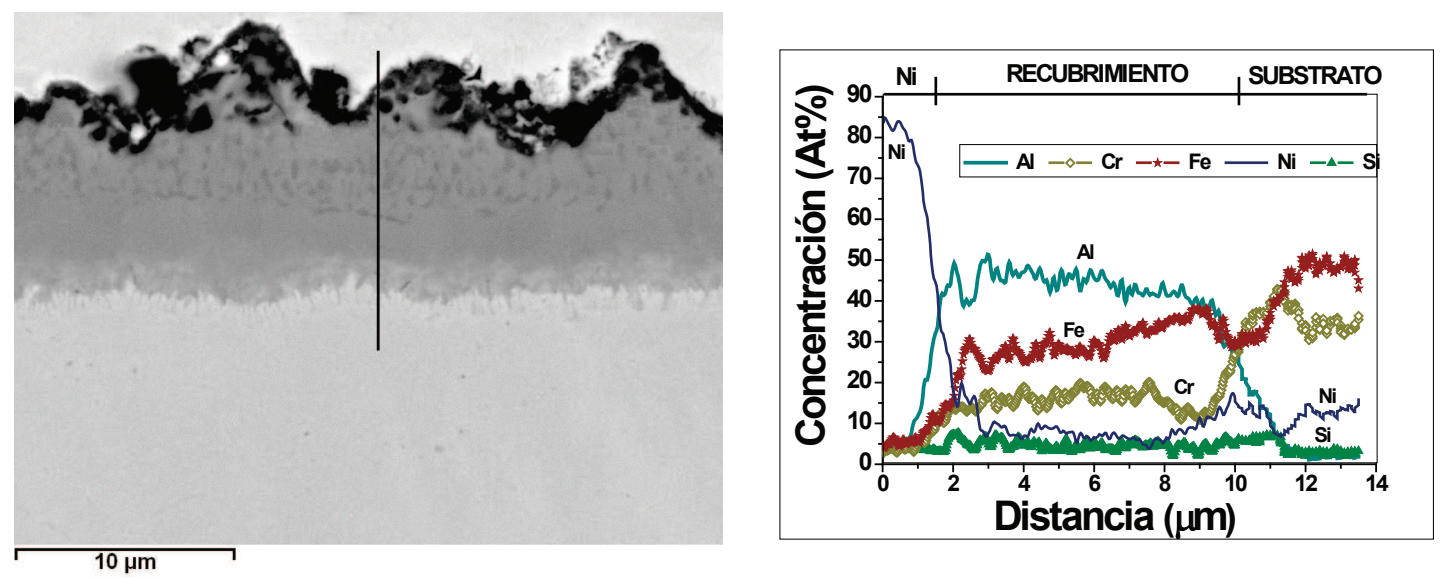

Fig. 3. Corte transversal del recubrimiento de aluminio-silicio con tratamiento térmico para el acero inoxidable austenítico AISI 316

El tratamiento térmico favoreció la transformación de las fases ricas en aluminio en otras con mayor contenido de hierro, con lo cual el recubrimiento mejora sus propiedades mecánicas y su resistencia a la corrosión $[4$, $9,17]$. Concluimos que por medio del proceso CVD-FBR se pueden obtener recubrimientos de aluminio-silicio de $10 \mu \mathrm{m}$ de espesor, para 
la temperatura de deposición de $540{ }^{\circ} \mathrm{C}$ y un tiempo de deposición de 1 hora. Después del tratamiento térmico estas capas pueden crecer hasta $14 \mu \mathrm{m}$ de espesor, y aparece una zona de interdifusión dentro del substrato. La simulación termodinámica es una buena herramienta para seleccionar las condiciones de trabajo y conocer los haluros de aluminio y silicio que están produciendo la deposición del revestimiento.

Se identificaron las fases presentes en los recubrimientos de aluminio-silicio depositados por CVD-FBR, por medio de difracción de rayos $\mathrm{X}$ (DRX) en el acero inoxidable austenítico AISI 316. Los principales picos fueron determinados utilizando los patrones de la JCPDS (Joint Comité on Powder Difraction Standards) [19]. En la Fig. 4 se observan los difractogramas de ángulo rasante para el acero AISI 316 recubierto con aluminiosilicio con y sin tratamiento térmico. Las fases presentes en el recubrimiento sin tratamiento térmico tienen una mayor cantidad de aluminio que el recubrimiento tratado térmicamente; además se aprecia el cambio de compuestos intermetálicos, ya que en el recubrimiento sin tratamiento térmico hay $\mathrm{Al}_{5} \mathrm{Fe}_{2}, \mathrm{FeAl}_{2}$, $\mathrm{Al}_{2} \mathrm{FeSi}, \mathrm{Cr}_{3} \mathrm{Si}, \mathrm{AlCrFe}_{2}$ y $\mathrm{Al}_{5} \mathrm{FeNi}$; después del tratamiento térmico desaparece el $\mathrm{Al}_{5} \mathrm{Fe}_{2} \mathrm{y}$ se forma el FeAl; además, como se observó en el corte transversal de este recubrimiento, se ve claramente que disminuyó el porcentaje de aluminio y se incrementan los porcentajes de hierro, cromo y níquel, por lo tanto, las fases que tienen estos elementos se vuelven más estables; esto corrobora el análisis de línea realizado por EDAX. Las especies sólidas y los compuestos que se formaron en el recubrimiento durante la deposición no fueron los mismos que aparecieron en la simulación termodinámica.

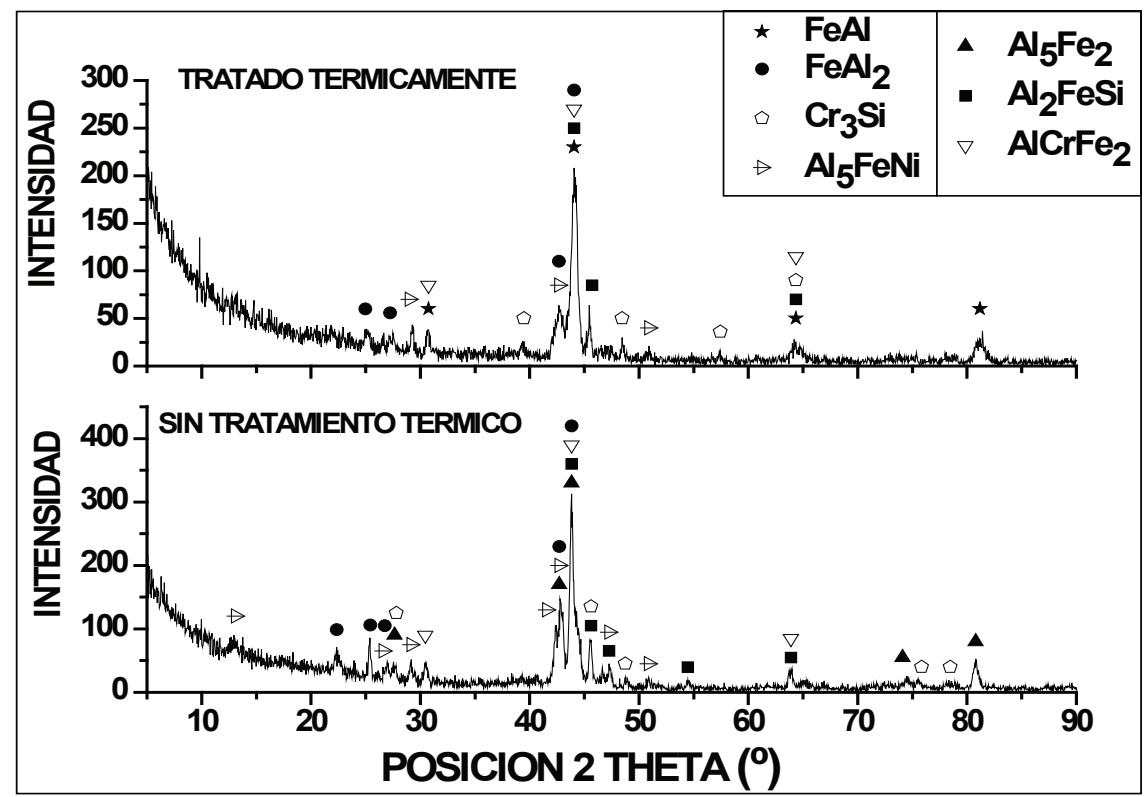

Fig. 4. Difractogramas de incidencia rasante para el acero AISI 316 recubierto con aluminio-silicio con y sin tratamiento térmico

\section{Conclusiones}

El estudio termodinámico a través de la simulación con el programa Thermo-Calc permitió un mejor ajuste de las condiciones experimentales de la deposición y una mejor comprensión de los fenómenos involucrados en la deposición de los recubrimientos; aunque los compuestos formados durante la simulación termodinámica no son iguales a los formados durante la deposición química de vapor en lecho fluidizado. 
Se mejoraron las condiciones de deposición de los recubrimientos de Al-Si por CVD-FBR a temperaturas por debajo de $600{ }^{\circ} \mathrm{C}$, la cual no afecta la microestructura de los aceros; además se obtuvieron espesores mayores a $15 \mu \mathrm{m}$ en los recubrimientos de aluminio-silicio después del tratamiento térmico. Además, la temperatura y el flujo de los gases precursores están directamente relacionados con la cinética de formación de los recubrimientos, en donde la temperatura de deposición de los recubrimientos no debe pasar de $560{ }^{\circ} \mathrm{C}$, porque se empiezan a clorinar los revestimientos y no se logran buenas capas.

En este trabajo se alcanzaron los mejores espesores para el recubrimiento aluminiosilicio a $540{ }^{\circ} \mathrm{C}$, durante 60 minutos y con una relación volumétrica de gases activos de $50 \%$ de argón, 46,83\% de hidrógeno y 3,17 \% de ácido clorhídrico, con un lecho de 2,5 g de aluminio, $7,5 \mathrm{~g}$ de silicio y $90 \mathrm{~g}$ de alúmina.

\section{REFERENCIAS}

[1] K. Oura, V. G. Lifshits, A. A. Saranin, A.V. Zotov y M. Katayama. Surface Science. Alemania, 2003.

[2] J. Marulanda, F. Pérez, S. Castañeda. Aluminum deposition on austenitic stainless steels AISI 304 and AISI 316 by FBCVD. Discussion meeting on the development of innovative iron aluminium alloys. Lanzarote-España, 2011.

[3] C. Kleijn, R. Dorsman, K. Kuijlaars, M. Okkerse, H. van Santen. Journal of Crystal Growth. 303 (2007), 362-380.

[4] S. Castañeda, F. Bolívar, F. Pérez. Oxidation of metal. 2010.

[5] L. Sánchez, F. Bolívar, M. Hierro, J. Trilleros, F. Pérez. Surface and Coatings Technology. 201 (2007), 7626-7634.

[6] J. Abella. Láminas delgadas y recubrimientos. Preparación, propiedades y aplicaciones. Consejo Superior de Investigaciones Científicas, CSIC. 2003.
[7] S. Castañeda, J. Marulanda, F. Pérez. Aluminide coatings on austenitic AISI $316 \mathrm{l}$ steel for protection of steam oxidation at high temperature: study of MS-TG. Discussion Meeting on the Development of Innovative Iron Aluminium Alloys. Lanzarote-España, 2011.

[8] N. Bahlawane. Thin Solid Films. 394 (2001), 298303.

[9] F. Pérez, F. Pedraza, M. Hierro, P. Hou. Surface and Coatings Technology. 133 (2000), 338-343.

[10] J. Pérez-Mariano, J. Caro, C. Colominas. Surface and Coatings Technology. 201 (2006), 4021-4025.

[11] L. Cadoret, N. Reuge, S. Pannala, M. Syamlal, C. Rossignol, J. Dexpert-Ghys, B. Caussat. Powder Technology 190 (2009), 185-191.

[12] S. Kouadri-Mostefa, P. Serp, M. Hémati, B. Caussat. Powder Technology 120 (2001), 82-87.

[13] L. Cadoret, N. Reuge, S. Pannala, M. Syamlal, C. Coufort, B. Caussat. Surface and Coatings Technology 201 (2007), 8919-8923.

[14] Thermocalc. Foundation of computational themodynamics, Stockholm (Sweden), 19952003.

[15] F. Bolívar, F. Pérez, M. Hierro, J. Trilleros, L. Sánchez. Scientia et Technica. 36 (2007), 619624.

[16] F. Pérez, S. Castañeda. Surface and Coatings Technology 201 (2007), 6239-6246.

[17] S. Majumdar, I.G. Sharma, S. Raveendra, I. Samajdar, P. Bhargava. Materials Science and Engineering A 492 (2008), 211-217.

[18] S. Majumdar, P. Sengupta, G. Kale, I. Sharma. Surface and Coatings Technology 200 (2006), 3713-3718.

[19] Standars Joint Committe on Powder Diffraction, JCPDS-ICDD (1997). 